\title{
DE MOIVRE-TYPE IDENTITIES FOR THE PADOVAN NUMBERS
}

\author{
Mücahit Akbıyık $^{\mathbf{a}^{*}}$ (D), Seda Yamaç Akbıyık ${ }^{\text {b }}$, Jeta Alo ${ }^{\mathbf{a}}$ \\ $a^{*}$ Department of Mathematics, Faculty of Art and Science., Beykent University, Istanbul, Turkey \\ mucahitakbiyik@beykent.edu.tr (*corresponding author) \\ ${ }^{b}$ Department of Computer Engineering, Faculty of Engineering and Architecture, Istanbul Gelisim \\ University, Istanbul, Turkey \\ syamac@gelisim.edu.tr, jeta@beykent.edu.tr
}

\begin{abstract}
At this work, we give a method for constructing the Perrin and Padovan sequences and obtain the De Moivre-type identity for Padovan numbers. Also, we define a Padovan sequence with new initial conditions and find some identities between all of these auxiliary sequences. Furthermore, we give quadratic approximations for these sequences.
\end{abstract}

Keywords: De Moivre-type identity, Padovan numbers, Perrin numbers, quadratic approximation

\section{Introduction}

According to Shannon [1], the Padovan numbers were first examined in 1924 by Gerard Cordonnier. Moreover, these numbers were independently restated by Dom Hans van der Laan with whom Padovan studied from 1980 until 1991. However, the sequence was written in the literature by Ian Stewart under the name Padovan. Stewart's Padovan numbers are defined by a recurrence relation $P_{n+3}=P_{n+1}+P_{n}$ with initial values $P_{0}=P_{1}=P_{2}=1$. Some terms of this sequence are:

\section{$1,1,1,2,2,3,4,5,7,9,12,16,21,28,37,49,65,86,114,151,200,265, \ldots$}

The Padovan sequence takes place in The On-Line Encyclopedia of Integer Sequences (OEIS) with the recurrence relation $P_{n+3}=P_{n+1}+P_{n}$, and the initial values $P_{0}=1, P_{1}=P_{2}=0$, (the sequence A000931 in [2]). Some terms of this sequence are: 
$1,0,0,1,0,1,1,1,2,2,3,4,5,7,9,12,16,21,28,37,49,65,86,114, \ldots$

In [3,1], the authors studied the Padovan numbers with the initial conditions $P_{0}=1, P_{1}=$ $0, P_{2}=1$. In [4], the authors, developed the matrix sequences that represent Padovan and Perrin numbers and presented some important relationships between Padovan and Perrin matrix sequences.

The characteristic equation of the Padovan Sequence is $x^{3}-x-1=0$. The real root of this characteristic equation is known as plastic ratio which was defined in 1924 by G'erard Cordonnier, [5].

In 1972, Bicknell and his friends presented the De Moivre-type identity for Fibonacci numbers, [6]. They showed that when $x_{1}=\frac{1+\sqrt{5}}{2}$ and $x_{2}=\frac{1-\sqrt{5}}{2}$ which are the roots of the characteristic equation of the Fibonacci numbers $x^{2}-x-1=0$, the following identity is satisfied

$$
((1 \pm \sqrt{5}) / 2)^{n}=\frac{L_{n} \pm \sqrt{5} F_{n}}{2}
$$

where $L_{n}$ denotes the $n$-th Lucas number and $F_{n}$ denotes the $n$-th Fibonacci number. The relation (1) is called De Moivre-type identity for Fibonacci numbers. After that, in 1988, Lin stated the De Moivre-type identity for the Tribonacci numbers (3-order Fibonacci ) by using the equation $x^{3}-x^{2}-x-1=0$, [7]. Also, he obtained the De Moivre-type identity for the tetranacci numbers whose characteristic equation is $x^{4}-x^{3}-x^{2}-x-1=0$, [8]. In [9, 10], the authors, gave the De Moivre-type identity for the Pell and Jacobsthal numbers. In [11], the author gave the quadratic approximations for generalized Tribonacci sequence.

In this paper, we give a way for constructing the Perrin and Padovan Sequences by using the roots of characteristic equation $x^{3}-x-1=0$. However, we obtain the De Moivre-type identity for Padovan numbers. We define a Padovan sequence with new initial conditions and find some identities between all of these auxiliary sequences. Also, we give quadratic approximations for these sequences.

\section{De Moivre-type identity for the Padovan Numbers}

The three roots of $x^{3}-x-1=0$ are

$$
\begin{aligned}
& r_{1}=\sqrt[3]{\frac{27+3 \sqrt{69}}{54}}+\sqrt[3]{\frac{27-3 \sqrt{69}}{54}} \\
& r_{2}=\omega \sqrt[3]{\frac{27+3 \sqrt{69}}{54}}+\omega^{2} \sqrt[3]{\frac{27-3 \sqrt{69}}{54}} \\
& r_{3}=\omega^{2} \sqrt[3]{\frac{27+3 \sqrt{69}}{54}}+\omega^{\frac{27-3 \sqrt{69}}{54}}
\end{aligned}
$$

where $\omega=-\frac{1}{2}+\frac{\sqrt{3}}{2} i$ is the primitive complex cube root of 1 . Moreover, these three roots can be written in the following forms:

$r_{1}=A+B$ 
$r_{2}=-\frac{1}{2}(A+B)+i \frac{\sqrt{3}}{2}(A-B)$,
$r_{3}=-\frac{1}{2}(A+B)-i \frac{\sqrt{3}}{2}(A-B)$,

where $A B=\frac{1}{3}$ and $A^{3}+B^{3}=1$. Thus, the powers of the root $r_{1}$ can be calculated as follows:

$r_{1}^{2}=\frac{2}{3}+\quad\left(A^{2}+B^{2}\right)$,

$r_{1}{ }^{3}=1+(A+B)$,

$r_{1}^{4}=\frac{2}{3}+(A+B)+\left(A^{2}+B^{2}\right)$,

$r_{1}^{5}=\frac{5}{3}+(A+B)+\left(A^{2}+B^{2}\right)$,

$r_{1}^{6}=\frac{5}{3}+2(A+B)+\left(A^{2}+B^{2}\right)$,

$r_{1}{ }^{7}=\frac{7}{3}+2(A+B)+2\left(A^{2}+B^{2}\right)$,

$r_{1}^{8}=\frac{10}{3}+3(A+B)+2\left(A^{2}+B^{2}\right)$.

The coefficients of the above equations construct the Padovan sequences which are denoted by $\left\{W_{n}\right\},\left\{Y_{n}\right\}$, respectively. Thus,

i. the sequence $\left\{W_{n}\right\}$ is the Perrin sequence with the recurrence relation $W_{n+3}=W_{n+1}+$ $W_{n}$ for $n \geq 3$ and $W_{0}=3, W_{1}=0, W_{2}=2$, [2].

ii. the sequence $\left\{Y_{n}\right\}$ is the Padovan sequence with the recurrence relation $Y_{n+3}=Y_{n+1}+$ $Y_{n}$ for $n \geq 3$ and $Y_{0}=1, Y_{1}=0, Y_{2}=1,[3,1]$.

We present some terms of the above sequences in the following table.

Table 1. Some terms of the sequences $\left\{\mathrm{W}_{\mathrm{n}}\right\},\left\{\mathrm{Y}_{\mathrm{n}}\right\}$ and $\left\{\mathrm{Z}_{\mathrm{n}}\right\}$

\begin{tabular}{|l|l|l|l|l|l|l|l|l|l|l|l|}
\hline$n$ & 0 & 1 & 2 & 3 & 4 & 5 & 6 & 7 & 8 & 9 & 10 \\
\hline$W_{n}$ & 3 & 0 & 2 & 3 & 2 & 5 & 5 & 7 & 10 & 12 & 17 \\
\hline$Y_{n}$ & 1 & 0 & 1 & 1 & 1 & 2 & 2 & 3 & 4 & 5 & 7 \\
\hline$Z_{n}$ & 2 & 1 & 0 & 3 & 1 & 3 & 4 & 4 & 7 & 8 & 11 \\
\hline
\end{tabular}

By using the sequences $\left\{W_{n}\right\},\left\{Y_{n}\right\}$ and applying induction over $n$, we construct the followings:

$r_{1}^{n}=\frac{1}{3} W_{n}+Y_{n-1}(A+B)+Y_{n-2}\left(A^{2}+B^{2}\right)$

Similarly, we get

$r_{2}^{n}=\frac{1}{3} W_{n}-\frac{1}{2}\left[Y_{n-1}(A+B)+Y_{n-2}\left(A^{2}+B^{2}\right)\right]+\frac{\sqrt{3}}{2} i\left[Y_{n-1}(A-B)-Y_{n-2}\left(A^{2}-B^{2}\right)\right]$,

and 
$r_{3}^{n}=\frac{1}{3} W_{n}-\frac{1}{2}\left[Y_{n-1}(A+B)+Y_{n-2}\left(A^{2}+B^{2}\right)\right]-\frac{\sqrt{3}}{2} i\left[Y_{n-1}(A-B)-Y_{n-2}\left(A^{2}-B^{2}\right)\right]$

where $W_{n}$ denotes the $n$-th term of $\left\{W_{n}\right\}$ and $Y_{n}$ denotes the $n$-th term of $\left\{Y_{n}\right\}$. Thus, we calculate the powers of the roots $r_{1}{ }^{n}, r_{2}{ }^{n}, r_{3}{ }^{n}$ in terms of $\left\{W_{n}\right\},\left\{Y_{n}\right\}$ which are called the De Moivretype identity for the Padovan numbers.

\section{Some properties of $\left\{W_{n}\right\},\left\{Y_{n}\right\}$ and $\left\{Z_{n}\right\}$}

In this section, some interesting identities are derived by using the definitions of the Padovan numbers. By using the recurrence relations of the sequences $\left\{W_{n}\right\},\left\{Y_{n}\right\}$, one can find from the recurrence relations that the following properties are satisfied:

- $W_{n+3}=3 Y_{n}+2 Y_{n+1}$

- $\sum_{k=0}^{n} W_{k}=3 Y_{n+2}+2 Y_{n+3}-2$

Now, we define the sequence $\left\{Z_{n}\right\}$ with the recurrence relation $Z_{n+3}=Y_{n+1}+W_{n}$ for $n \geq 3$ and with the initial values $Z_{0}=2, Z_{1}=1, Z_{2}=0$. Note that the sequence $\left\{Z_{n}\right\}$ is also a Padovan sequence with the recurrence relation $Z_{n+3}=Z_{n+1}+Z_{n}$ for $n \geq 3$ and $Z_{0}=2$, $Z_{1}=1, Z_{2}=0$. First terms of the sequence $\left\{Z_{n}\right\}$ are presented in the Table 1 . Also, the following identities can be obtained for this sequence:

- $Z_{n+3}=2 Y_{n}+Y_{n+2}$

- $\sum_{k=0}^{n} Z_{k}=2 Y_{n+2}+Y_{n+4}-1$

- $\sum_{k=0}^{n} Z_{2 k}=Z_{2 n+3}-1$

- $\quad \sum_{k=0}^{n} Z_{2 k+1}=Z_{2 n+4}$

- $\sum_{k=0}^{n} Z_{3 k}=Z_{3 n+2}+2$

- $\quad \sum_{k=0}^{n} Z_{3 k+1}=Z_{3 n+3}-2$

- $\sum_{k=0}^{n} Z_{3 k+2}=Z_{3 n+4}-1$

- $\sum_{k=0}^{n} Z_{5 k}=Z_{5 n+1}+1$

Some of the proofs above can be found easily as follows:

From the recurrence relation $Z_{n}=Z_{n+3}-Z_{n+1}$, we find

$$
\begin{gathered}
Z_{0}=Z_{3}-Z_{1} \\
Z_{1}=Z_{4}-Z_{2} \\
Z_{2}=Z_{5}-Z_{3} \\
\quad \cdots \\
Z_{n-1}=Z_{n+2}-Z_{n} . \\
Z_{n}=Z_{n+3}-Z_{n+1}
\end{gathered}
$$

By summing side by side and using the equality $Z_{n+3}=2 Y_{n}+Y_{n+2}$, we get

$$
\sum_{k=0}^{n} Z_{k}=Z_{n+5}-1=2 Y_{n+2}+Y_{n+4}-1 \text {. }
$$

Similarly, we have 


$$
\begin{aligned}
& Z_{0}=Z_{3}-Z_{1} \\
& Z_{2}=Z_{5}-Z_{3} \\
& \quad \cdots \\
& Z_{2 n-2}=Z_{2 n+1}-Z_{2 n-1} \\
& Z_{2 n}=Z_{2 n+3}-Z_{2 n+1} .
\end{aligned}
$$

By summing side by side we get

$$
\sum_{k=0}^{n} Z_{2 k}=Z_{2 n+3}-Z_{1}=Z_{2 n+3}-1 .
$$

The other all proofs can be easily found by similar methods.

Moreover, we calculate the quadratic approximations of the sequences $\left\{W_{n}\right\},\left\{Y_{n}\right\}$ and $\left\{Z_{n}\right\}$. The following results can be easily found directly with the help of the reference [11].

- Quadratic approximation of $\left\{W_{n}\right\} \Rightarrow\left\{\begin{array}{l}K_{1} r_{1}{ }^{n+2}=W_{n+2} r_{1}{ }^{2}+W_{n+1} r_{1}+W_{n} r_{1}+W_{n-1} \\ L_{1} r_{2}{ }^{n+2}=W_{n+2} r_{2}{ }^{2}+W_{n+1} r_{2}+W_{n} r_{2}+W_{n-1} \\ M_{1} r_{3}{ }^{n+2}=W_{n} r_{3}{ }^{2}+W_{n+1} r_{3}+W_{n} r_{3}+W_{n-1}\end{array}\right.$

where $K_{1}=2-3 r_{2} r_{3} \approx-0.2649, \quad L_{1}=2-3 r_{1} r_{3} \approx 4.6324+2.2346 i$, and $M_{1}=2-$ $3 r_{1} r_{2} \approx 4.6324-2.2346 i$.

- Quadratic approximation of $\left\{Y_{n}\right\} \Rightarrow\left\{\begin{array}{l}K_{2} r_{1}^{n+2}=Y_{n+2} r_{1}{ }^{2}+Y_{n+1} r_{1}+Y_{n} r_{1}+Y_{n-1} \\ L_{2} r_{2}{ }^{n+2}=Y_{n+2} r_{2}{ }^{2}+Y_{n+1} r_{2}+Y_{n} r_{2}+Y_{n-1} \\ M_{2} r_{3}{ }^{n+2}=Y_{n+2} r_{3}{ }^{2}+Y_{n+1} r_{3}+Y_{n} r_{3}+Y_{n-1}\end{array}\right.$

where $K_{2}=1-r_{2} r_{3} \approx 0.2450, L_{2}=1-r_{1} r_{3} \approx 1.8775+0.7449 i$, and $M_{2}=1-r_{1} r_{2} \approx$ $1.8775-0.7449 i$.

- Quadratic approximation of $\left\{Z_{n}\right\} \Rightarrow\left\{\begin{array}{l}K_{3} r_{1}{ }^{n}=Z_{n+2} r_{1}{ }^{2}+Z_{n+1} r_{1}+Z_{n} r_{1}+Z_{n-1} \\ L_{3} r_{2}{ }^{n}=Z_{n+2} r_{2}{ }^{2}+Z_{n+1} r_{2}+Z_{n} r_{2}+Z_{n-1} \\ M_{3} r_{3}{ }^{n}=Z_{n+2} r_{3}{ }^{2}+Z_{n+1} r_{3}+Z_{n} r_{3}+Z_{n-1}\end{array}\right.$

where $K_{3}=-\left(r_{2}+r_{3}\right)+2 r_{2} r_{3} \approx 2.8347, L_{3}=-\left(r_{1}+r_{3}\right)+2 r_{1} r_{3} \approx-2.4173-0.9275 i$, and $M_{3}=-\left(r_{1}+r_{2}\right)+2 r_{1} r_{2} \approx-2.4173+0.9275 i$.

\section{Conclusion}

In this paper, we present a way for the construction of Padovan and Perrin sequences similar to that of Fibonacci and Lucas sequences. In addition, we give the De Moivre-type identity for the Padovan numbers. Also, we show that there are also parallels between the Fibonacci numbers $F_{n}$, and the Padovan numbers $Y_{n}$, such as

$x_{1}{ }^{n}=\frac{L_{n}+\sqrt{5} F_{n}}{2}$,

$x_{2}^{n}=\frac{L_{n}-\sqrt{5} F_{n}}{2}$,

and 


$$
\begin{aligned}
& r_{1}^{n}=\frac{1}{3} W_{n}+Y_{n-1}(A+B)+Y_{n-2}\left(A^{2}+B^{2}\right), \\
& r_{2}^{n}=\frac{1}{3} W_{n}-\frac{1}{2}\left[Y_{n-1}(A+B)+Y_{n-2}\left(A^{2}+B^{2}\right)\right]+\frac{\sqrt{3}}{2} i\left[Y_{n-1}(A-B)-Y_{n-2}\left(A^{2}-B^{2}\right)\right], \\
& r_{3}^{n}=\frac{1}{3} W_{n}-\frac{1}{2}\left[Y_{n-1}(A+B)+Y_{n-2}\left(A^{2}+B^{2}\right)\right]-\frac{\sqrt{3}}{2} i\left[Y_{n-1}(A-B)-Y_{n-2}\left(A^{2}-B^{2}\right)\right],
\end{aligned}
$$

in which $x_{1}, x_{2}$ and $r_{1}, r_{2}, r_{3}$ are the roots of the corresponding characteristic equations. Similar parallels exist between the Lucas numbers $L_{n}$ and the Perrin numbers $W_{n}$, too. That is, the Perrin sequence can be thought of as the Lucas sequence of the Padovan sequence. Finally, we define a Padovan sequence with new initial conditions which is obtained from Padovan and Perrin numbers. We give quadratic approximations and some identities for these three sequences.

\section{Conflicts of interest}

No conflict of interest was declared by the authors.

\section{References}

[1] Shannon, A. G., Anderson, P. G. , Horadam, A. F., "Properties of Cordonnier, Perrin and Van der Laan numbers”, International Journal of Mathematical Education in Science and Technology 37(7) (2006) : 825-831.

[2] Sloane, N.J.A., “The on-line encyclopedia integer sequences” , http://oeis.org/. Access date: 10.03.2021.

[3] Vieira, R. P. M., Alves, F. R. V., Cruz, P. M. M., "Catarino Padovan sequence generalization -a study of matrix and generating function”, Notes on Number Theory and Discrete Mathematics 26(4) (2020) : 154-163.

[4] Yilmaz, N., Taskara, N., "Matrix Sequences in terms of Padovan and Perrin Numbers”, Journal of Applied Mathematics (2013) : 1-7.

[5] Yilmaz, N., Taskara, N., "Binomial Transforms of the Padovan and Perrin Matrix Sequences”, Abstract and Applied Analysis (2013) : 1-7.

[6] Basin, S. L., "Elementary problems and solutions”, Fibonacci Q. 1 (1963) : 77.

[7] Lin, P.Y., "De Moivre-Type Identities for the Tribonacci Numbers”, The Fibonacci Quarterly 26(2) (1988) : 131-134.

[8] Lin, P.Y., “De Moivre-Type Identities for the Tetranacci Numbers”, In: Bergum G.E., Philippou A.N., Horadam A.F. (eds) Applications of Fibonacci Numbers. Springer, Dordrecht, 4 (1991) : 215-218.

[9] Yamaç Akbıyık, S., Akbıyı, M., "De Moivre-Type Identities for the Pell Numbers”, Turkish Journal of Mathematics and Computer Science 13 (1) (2021) : 63-67.

[10] Akbıyık, M., Yamaç Akbıyık, S., "De Moivre-Type Identities for the Jacobsthal Numbers”, Notes on Number Theory and Discrete Mathematics 27 (3) (2021) : 95-103.

[11] Cerda-Morales, G., "Quadratic Approximation of Generalized Tribonacci Sequences”, Discussiones Mathematicae General Algebra and Applications 38 (2018) : 227-237. 